\title{
A token-actuated timer for line-voltage devices
}

\author{
M. DURBIN and L. A. JASON \\ DePaul University, Chicago, Illinois
}

\begin{abstract}
A token-actuated timer that detects the insertion of a flat washer ("token") is described. The unit provides a timed line-voltage output and counts the number of times the line-voltage device has been operated. Using behavioral token economies, this device has been used to control the television watching of children by monitoring television watching time, and by controlling the amount of time the television is on.
\end{abstract}

Many studies indicate that most youngsters watch a considerable amount of television; therefore, there is a need to better monitor and control television viewing. By age 18 years, the average American child has spent more time watching television than engaging in any other activity except sleep (Lesser, 1970). An alarming finding is that there appears to be a moderate relationship between watching television and subsequent aggressive behavior (Lefkowitz, Eron, Walder, \& Huesmann, 1977). Also, increased television watching may be linked to lower academic achievement (Rubenstein, 1983).

These findings suggest a need to develop a monitoring system to record the amount of time spent watching television, as well as to control the amount of time that the television set is on. The system described here can be used by investigators, using behavioral token economies, to control the television watching of children.

\section{TIMER}

Essentially, the token-actuated timer is a monostable multivibrator, that is, a one-shot pulse generator, with an output pulse width of $30 \mathrm{~min}$. If a valid token is detected, then a 30-min timer is actuated and a relay is energized, thereby connecting the television set to the house line current. The timer consists of three subsec. tions: token detection, timer and relay control, and token counting.

\section{Circuit Description}

Token detection. The function of the token-detection section is to ascertain when a valid token has been inserted and, consequently, to trigger the 30-min timer and increment the token counter. A schematic diagram is provided in Figure 1. The tokens are standard 5/16-in. flat washers with an outer diameter of $3 / 4 \mathrm{in}$. and an inner diameter of $9 / 32$ in. The detector assembly consists of an XC-880A infrared-emitting diode and a TIL414 infrared phototransistor. These components are mounted at the bottom of a rectangular shaft that is approxi-

Requests for reprints should be sent to: Martin J. Durbin, DePaul University, Department of Physics, 2219 N. Kenmore Avenue, Chicago, IL 60614. mately $1 \mathrm{in.}$ long. The shaft is bolted to the top of the timer chassis, in which a hole that is equal to the width of the tokens has been milled out. Thus, to turn on the television, the subject deposits a token, which falls past the detector assembly into a catch box below. A valid token is distinguished by the amount of time it takes to pass the infrared detector and by the hole in its center.

A token is verified when the infrared phototransistor is turned off twice by the passage of the token within an alloted time interval. As shown in Figure 1, the sequence of events is as follows. When the leading edge of the falling token passes the phototransistor, the JK flipflop, U1, is toggled, thereby triggering one-shots U3 and U4. The output pulse width for one-shot U3 is approximately $3.2 \mathrm{msec}$, which is about one-third of the time it takes the leading rim of the token to pass the detector. When U3 times out, counter U7 is incremented to 1 and the JK flip-flop is cleared to await another trigger. The trailing rim of the token then triggers $\mathrm{U} 1$, which in turn retriggers $\mathrm{U} 3$. When $\mathrm{U} 3$ times out, it increments $\mathrm{U} 7$ to a count of 2 , which causes its $q_{1}$ output to go high. When a valid token has been inserted, one-shot $\mathrm{U} 4$, which has an output pulse width of $97-\mathrm{msec}$, does not time out, and consequently the 30-min timer, U6, is triggered.

Timer and relay control. The $30-\mathrm{min}$ timer is U6, a UA2240 timer-counter configured as a triggered, astable multivibrator. The free-running frequency of $\mathrm{U} 6$ is approximately $0.07 \mathrm{~Hz}$, which is divided by 128 at its $q_{7}$ output to provide a pulse width of $1,800 \mathrm{sec}$. Shorter pulse widths can be formed by selecting one of the other counter outputs of U6. For example, by using $\mathrm{q}_{6}$ (pin 7), an output pulse width of $15 \mathrm{~min}$ can be formed. Different free-running frequencies can be achieved by changing the RC time constant set by pin 13. After a valid token has been detected, the output of U6, TVON', goes low. This disables one-shot U2 and causes the phototransistor within the optoisolator shown in Figure 2 to conduct. Thus, the gate of the power FET shown in Figure 3 is brought high, causing it to conduct and thereby energizing the relay coil. This causes the relay contacts to close, thereby connecting the television set to the house line current.

Token counting. The token counter comprises cas- 


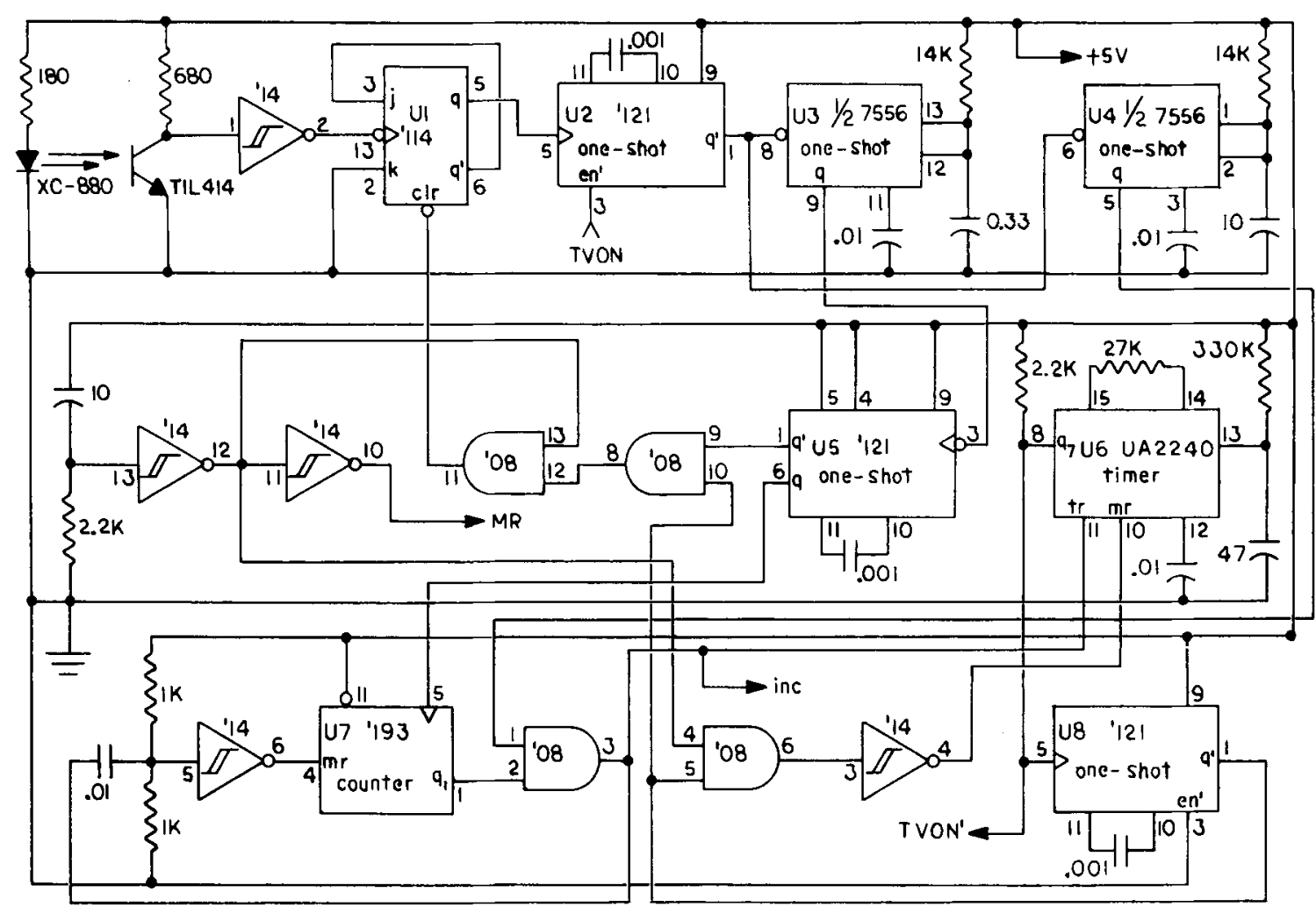

Figure 1. Token detection circuit.

caded 4-bit binary counters U9 and U10, shown in Figure 2. Each time the $30-\mathrm{min}$ timer U6 is triggered, the token counter is also incremented. The token count is displayed in an inverted binary format via the eight LEDs connected to the outputs of U9 and U10. This display representation was chosen both for its simplicity in design and for its ability to yield an independent confirmation of the number of tokens used. The token counter is reset when the unit is first plugged in via the master reset signal, MR.

\section{Construction}

The components for the token-actuated timer are housed within a standard aluminum chassis box. The front of the box has a large hole milled out to accept a smaller box used to catch the tokens. This catch box has a key lock fitted onto it. The house line current for the television is brought out via a female plug that is contained in another box that has a lockable hinged lid. This means that a subject cannot unplug the television and replug it into a standard wall outlet. The lower end of the detector shaft assembly is terminated in a hook constructed from stiff drawn wire. This hook prevents a child from tying a string to a token and then repeatedly lowering and raising that token. A number of measures were taken in the design of the token-actuated timer to prevent transients, due to relay switching, from feeding back to the logic circuit. As seen in Fig-

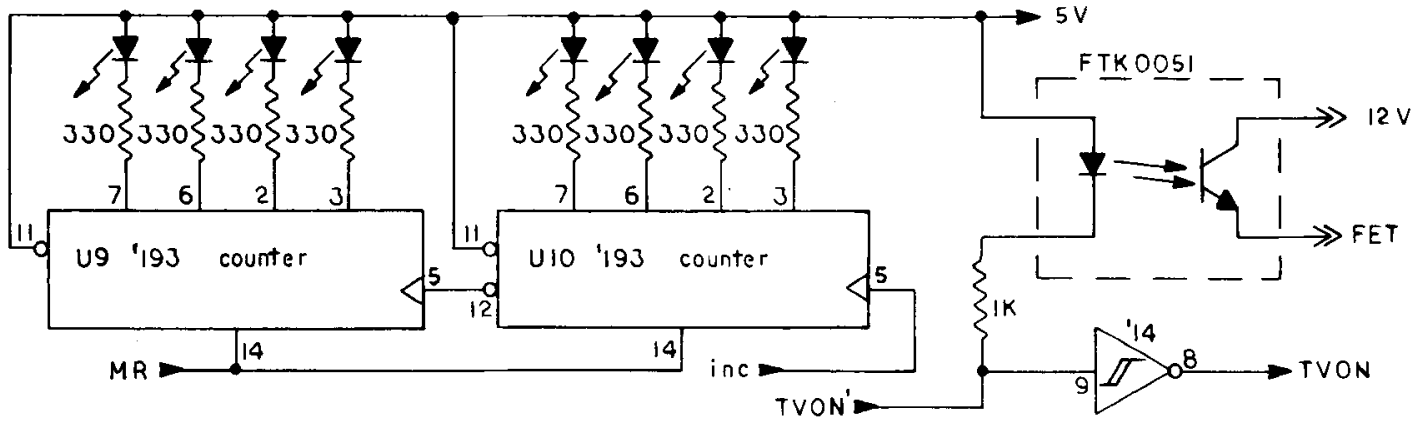

Figure 2 Token counter and relay optoisolator. 


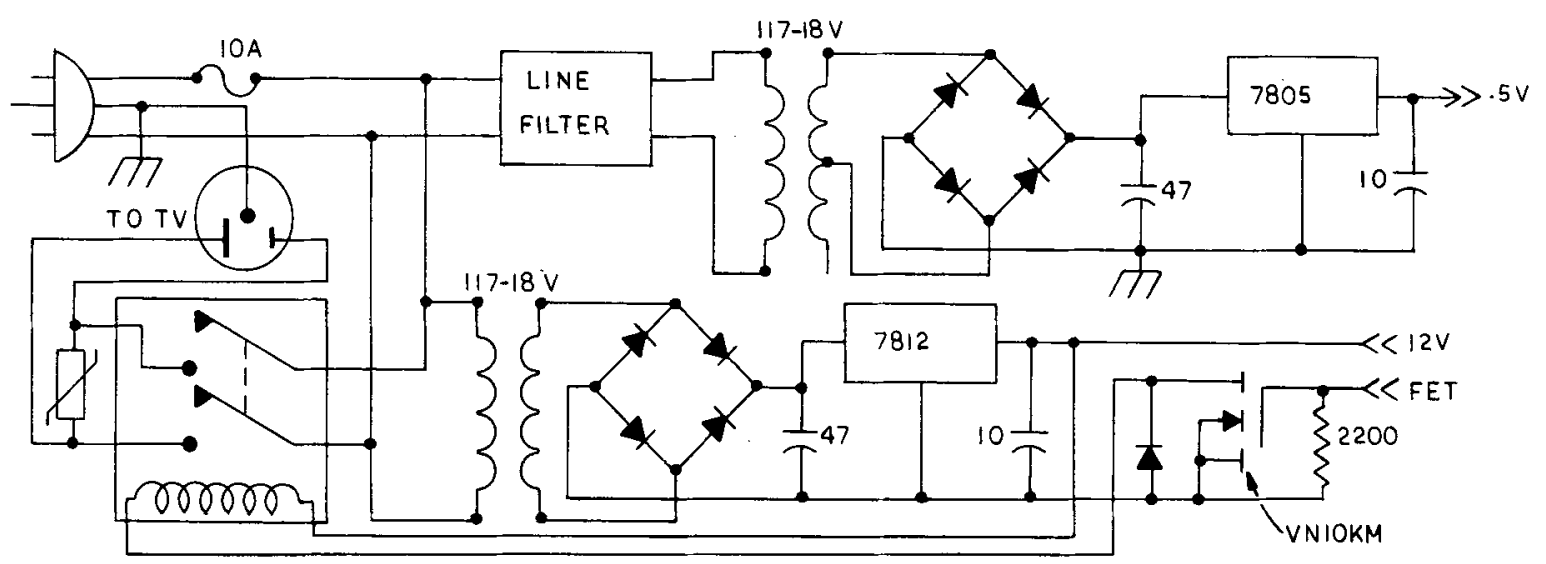

Figure 3. Power supplies and relay driver.

ure 3 , these measures included the use of an RFI line filter on the $5-\mathrm{V}$ power supply, complete separation of the relay and logic circuits via separate power supplies and an optoisolator (shown in Figure 2), and clamping of transients onto the television side of the relay by a metal oxide varistor.

The estimated construction cost for the tokenactuated timer is $\$ 50$.

\section{SUMMARY}

The token-actuated timer can provide a reliable count of the number of hours a television is on daily and implement a token economy to reduce television viewing time for children who are excessive watchers. A study by Jason and Rooney-Rebeck (in press) used the token timer with a youngster evidencing high levels of television viewing behavior. Their study employed a modified token economy in which earned tokens were used to activate the television for set periods of time.
With onset of intervention, immediate and dramatic reductions in television viewing were noted. The tokenactuated timer can also be used with other line-voltage devices, such as computers, video games, stereos, etc.

\section{REFERENCES}

Jason, L. A., \& Rooney-Rebeck, P. (in press). Reducing excessive television watching. Child and Family Behavior Therapy. Lefkowitz, N. N., Eron, L. D., Walder, L. D., \& HuesmanN, R. L. (1977). Growing up to be violent: $A$ longitudinal study of the development of aggression. New York: Pergamon Press.

LESSER, G. S. (1970). Designing a program for broadcast television. In F. F. Korten, S. W. Cook, \& G. L. Lacey (Eds.), Psychology and the problems of society. Washington, DC: American Psychological Association.

Rubenstein, E. A. (1983). Television and behavior: Research conclusions of the 1982 NIMH report and their policy implications. American Psychologist, 38, 820-825.

(Manuscript received March 9, 1984; revision accepted for publication May $8,1984$. 